\title{
Effects of nanofiller morphology and aspect ratio on the rheo-mechanical properties of polyimide nanocomposites
}

\author{
V. E. Yudin 1 , J. U. Otaigbe ${ }^{2 *}$, V. M. Svetlichnyi ${ }^{1}$, E. N. Korytkova ${ }^{3}$, O. V. Almjasheva ${ }^{3}$, \\ V. V. Gusarov ${ }^{3}$ \\ ${ }^{1}$ Institute of Macromolecular Compounds, Russian Academy of Science, St.Petersburg, RUSSIA \\ ${ }^{2}$ School of Polymers and High Performance Materials, The University of Southern Mississippi, Hattiesburg, \\ MS 39406, USA \\ ${ }^{3}$ Institute of Silicate Chemistry, Russian Academy of Sciences, St.Petersburg, RUSSIA
}

Received 19 March 2008; accepted in revised form 2 May 2008

\begin{abstract}
New polyimide nanocomposites containing organically modified montmorillonite (MMT), synthetic silicate (chrysotile) nanotubes (SNT), and zirconium dioxide $\left(\mathrm{ZrO}_{2}\right)$ were prepared to investigate the influence of the nanoparticle morphology on the nanocomposite rheology and mechanical properties under selected conditions that the materials are likely to encounter during use. The efficiency of homogeneous dispersion of the nanoparticles in the polyimide matrix was studied by measuring the rheology of model oligoimides (OI) dispersions containing the desired amounts of the nanoparticles. The OI/nanoparticles dispersions showed significant increase in complex viscosity with increasing concentration of the nanoparticles that depended strongly on the nanoparticle morphology and aspect ratio. Polyimide nanocomposite films (PI-PM) prepared from the poly(amic acid) of poly(pyromellitic dianhydride-co-4,4'-oxydianiline) (PM) filled with the desired concentration of the nanoparticles showed an increase in tensile modulus with increasing nanoparticle concentration in the order MMT $>\mathrm{SNT}>\mathrm{ZrO}_{2}$. In contrast to the PI-PM/MMT films, the PI-PM films filled with 10 vol\% of SNT and $\mathrm{ZrO}_{2}$ showed higher sample failure strains, suggesting that the SNT and $\mathrm{ZrO}_{2}$ may be more effective in improving the ductility of the polyimide nanocomposites for applications where the relatively brittle polyimide/MMT nanocomposites films are not useable.
\end{abstract}

Keywords: nanocomposites, polyimide, ceramic nanotubes and nanoparticles, rheology, mechanical properties

\section{Introduction}

Organic polymer nanocomposites containing small amounts of various inorganic nanoparticles where the size of the inorganic phase does not exceed one hundred nanometers offer a number of desirable characteristics in a number of applications [1]. It is worthy to note that optimal combinations of organic (polymer) and inorganic (nanofiller) can lead to functional polymer nanocomposites with new polymer structures and enhanced properties including thermal, mechanical, and electrical properties.
Nanocomposites are commonly based on polymer matrices reinforced with layered silicate nanofillers such as montmorillonite clay (MMT) with layer thickness of at least $1 \mathrm{~nm}[2,3]$. Polymer/MMT nanocomposites constitute a relatively new class of reinforced plastics prepared by uniformly dispersing MMT nanoparticles in a polymer matrix. Such nanocomposites have great scientific and practical importance due to their high mechanical and barrier properties, as well as, their fire resistance [4]. But MMT nanoparticles are well known to be a mixture of several natural compounds with nonuniform

*Corresponding author, e-mail: Joshua.Otaigbe@ usm.edu

(c) BME-PT and GTE 
composition and particle size. For example, MMT minerals from different deposits might differ considerably in composition. This variation in composition of MMT significantly complicates the task of making functional nanocomposites with prescribed properties for targeted applications. Therefore, there is a need to develop synthetic nanofillers with prescribed particle composition, shape and size for use as fillers in polymer nanocomposites with well defined properties.

In this area, synthetic nano-dimensional silicates may provide a number of opportunities in polymer nanocomposites that is relatively little studied and poorly understood relative to the well studied polymer nanocomposites filled with natural layered MMTs [5-9]. In contrast to the commonly used layered MMT compounds, it is envisaged that use of nanoparticles with different morphology (e.g., nanotubes and nanoparticles of isometric shape) might provide additional benefits in polymer nanocomposites not possible with MMT. Suitable techniques for hydrothermal synthesis of silicate nanotubes and particles of isometric form (e.g., zirconium dioxide) that gives the possibility to control the resulting nanoparticle's structure by varying the mode of hydrothermal synthesis has been reported [7-9].

Polyimide (PI)-based nanocomposites are of special interest because of the well known excellent heat resistance, chemical stability, and superior electric properties of polyimides [10]. As previously reported, PI/MMT nanocomposites can exhibit increased modulus and strength, high heat distortion temperature, decreased thermal expansion coefficient, reduced gas permeability, and increased solvent resistance compared to the pristine polymer $[2,3]$. However, there are a number of significant limitations in the processing of PI/MMT nanocomposites. These limitations include (but not limited to) the relatively low thermal stability of the organically modified MMT under typical processing temperatures compared to that of the relatively elevated processing temperatures that are needed for curing PIs or melt blending PIs with nanoparticles. It is well known that it is very difficult to find thermally stable modifier to improve clay compatibility with high temperature polymers such as polyimides that must be processed at elevated temperatures as already mentioned.

The work presented herein is focused on investigating the feasibility of using as nanofillers for polyimide matrices new silicate type nanotubes (SNT) and zirconium dioxide $\left(\mathrm{ZrO}_{2}\right)$ nanoparticles (with different morphology as compared to layered MMT platelets) that were custom synthesized specifically for nanocomposite application [7, 8]. The rheology and mechanical behavior of the PI nanocomposites as a function of the nanofiller shapes (i.e., platelets, nanotubes and isometric form) will be presented and discussed to provide a starting point for further experiments that will improve our understanding of optimal methods of incorporating nanoparticles with varying shapes and sizes into polyimide matrices and of the effects of the nanoparticles morphology on the structure and properties of polyimide nanocomposites in general.

\section{Experimental}

\subsection{Silicate nanotubes}

Silicate nanotubes (SNT) with a chrysotile type structure of $\mathrm{Mg}_{3} \mathrm{Si}_{2} \mathrm{O}_{5}(\mathrm{OH})_{4}$ were synthesized using a hydrothermal method that is facilitated by high pressure autoclaves as described elsewhere [7]. The SNT density is $2.5 \mathrm{~g} / \mathrm{cm}^{3}$. TEM showed that the SNT particles have average outer diame-

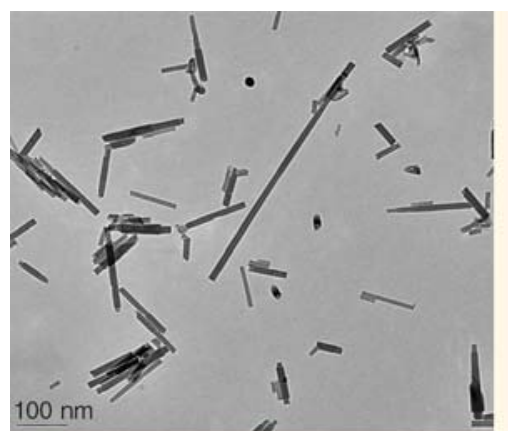

a)

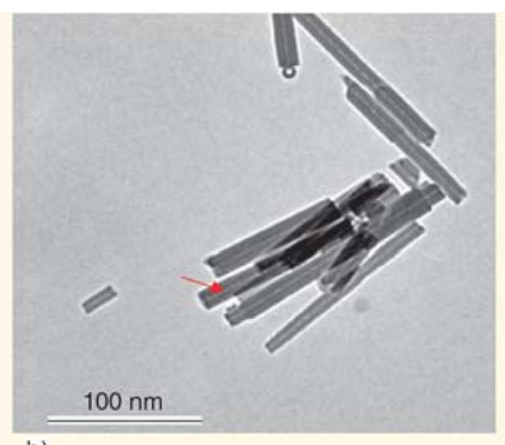

b)

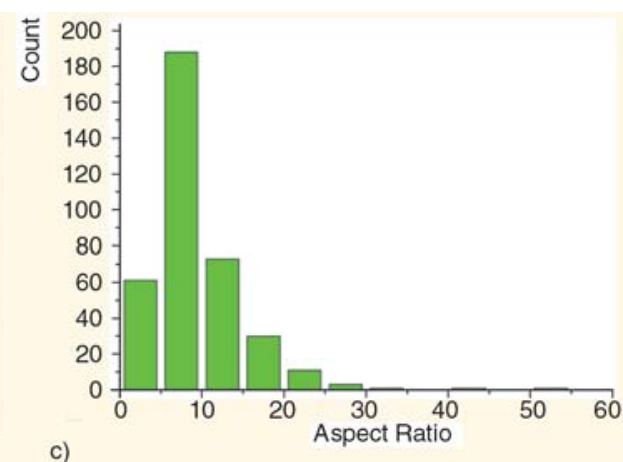

c)

Figure 1. TEM photomicrographs of the SNT particles at low (a) and high (b) magnifications; (c) frequency count of aspect ratio (length/diameter) of the SNT particles 
ter $=15 \mathrm{~nm}$ and average interior diameter $=3 \mathrm{~nm}$ as illustrated in Figures 1a and 1b. The length of the SNT ranges from 50 to $600 \mathrm{~nm}$ and its average aspect ratio is 10 (Figure 1c).

\subsection{Zirconium dioxide particles}

Zirconium dioxide $\left(\mathrm{ZrO}_{2}\right)$ particles were synthesized using a hydrothermal method [8]. These particles have spherical shapes as indicated in Figure 2. The average diameter of these particles is $20 \mathrm{~nm}$. The density of the particles is $\sim 6 \mathrm{~g} / \mathrm{cm}^{3}$.

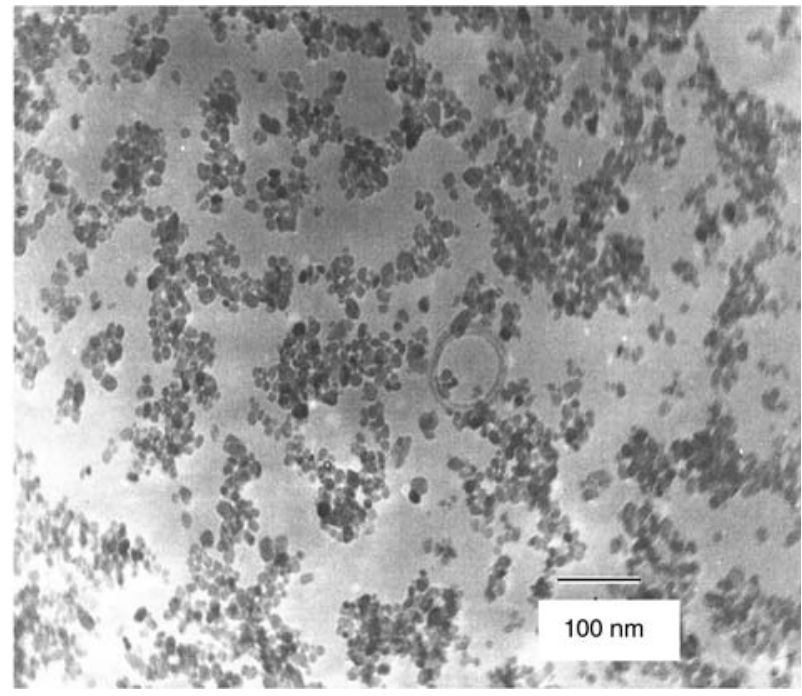

Figure 2. TEM photomicrograph of $\mathrm{ZrO}_{2}$ type particles

\subsection{Treatment of SNT and $\mathrm{ZrO}_{2}$ with silane coupling agent}

To improve compatibility of the nanoparticles with polyimide matrix, the $\mathrm{SNT}$ and $\mathrm{ZrO}_{2}$ particles were treated with $m$-aminophenyltrimethoxysilane (Gelest. Inc.) following the procedure described in [11]. The amount of the silane needed to obtain minimum uniform multilayer coverage was estimated from the known values of the specific wetting surface of the silane (i.e., $\sim 350 \mathrm{~m}^{2} / \mathrm{g}$ ) and the surface area of the filler (i.e., $\sim 100 \mathrm{~m}^{2} / \mathrm{g}$ ) and found to be about 3.5 times less than the amount of filler used. Specifically, one gram of nanoparticles (SNT or $\mathrm{ZrO}_{2}$ ) was dispersed in $50 \mathrm{ml}$ of ethanol with the aid of an ultrasonic mixer $(40 \mathrm{kHz}$, average sonic power: $45 \mathrm{~W}$ ) for 1 hour. $0.3 \mathrm{ml}$ of the silane was added to the sonicated suspension followed by additional sonication for $10 \mathrm{~min}$. The resulting suspension of the nanoparticles was centrifuged and the supernatant ethanol was decanted to yield the

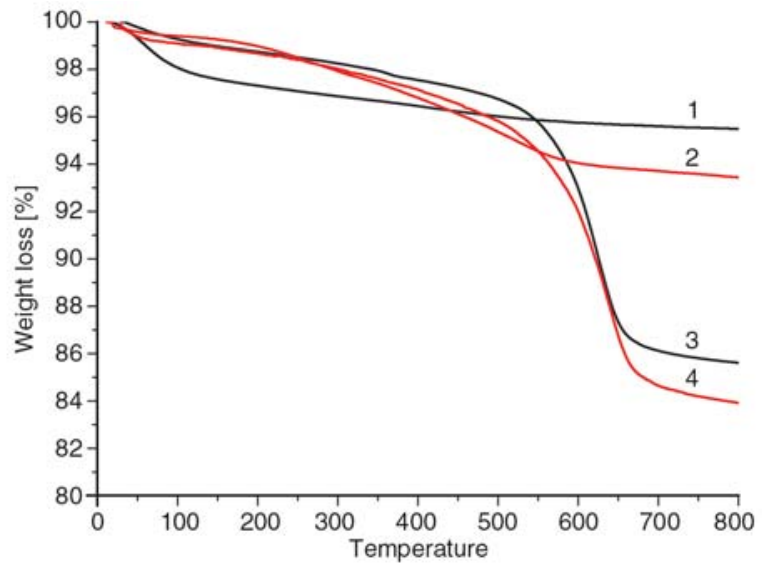

Figure 3. TGA scans of nanoparticles: 1 - pure $\mathrm{ZrO}_{2}$ nanoparticles; $2-\mathrm{ZrO}_{2}$ treated with silane coupling agent; 3 - pure SNT type particles; 4 - SNT treated with silane coupling agent

silane-treated nanoparticles of this study. The silane-treated nanoparticles were rinsed twice with ethanol followed by curing of the silane layer and dried until constant weight was achieved in a vacuum oven maintained at $60^{\circ} \mathrm{C}$ for $5 \mathrm{hr}$.

TGA was used to confirm the silane treatment of the SNT and $\mathrm{ZrO}_{2}$ as depicted in Figure 3. This figure shows degradation temperature of the organosilane compound at temperatures greater than $300^{\circ} \mathrm{C}$, making the silane-treated particles suitable for processing/mixing with polyimide matrices that must be cured at temperatures ranging from 200 to $300^{\circ} \mathrm{C}$. The weight loss of pure SNT particles at $550-650^{\circ} \mathrm{C}$ (Figure 3 ) is ascribed to evaporation of water incorporated into the crystal structure of chrysotile.

\subsection{Organic treatment of MMT}

Natural montmorillonite clay Na-MMT $\left[\right.$ Cloisite ${ }^{\circledR} \mathrm{Na}^{+}$, cation exchange capacity (CEC): $92.6 \mathrm{meq} / 100 \mathrm{~g}$ ] was obtained from Southern Clay Products, Inc. The density of the particles is $\sim 2.8 \mathrm{~g} / \mathrm{cm}^{3}$. Hydrochloric acid (concentration: $36.5 \%$ ) and $4,4^{\prime}$ bis (4"-aminophenoxy) diphenylsulfone (BAPS) were obtained from Fisher Chemical and Wakayama Seika Kogyo Co., Ltd. in Japan, respectively.

The Na-MMT was organically modified with BAPS ammonium salts. A mixture of BAPS and hydrochloric acid in deionized water (DI water) was prepared. An aqueous dispersion of Na-MMT that was agitated for 3 hours was added to this pre- 
viously prepared mixture just mentioned. The resulting mixture was agitated simultaneously with a mechanical stirrer and ultrasound at $60^{\circ} \mathrm{C}$ for 6 hours, and the mixture was then left at room temperature for 12 hours. The resulting white precipitate was filtered and washed repeatedly with DI water at $60^{\circ} \mathrm{C}$ to remove superfluous ammonium salts and $\mathrm{Cl}^{-}$ion. Removal of $\mathrm{Cl}^{-}$ion was monitored by titration with addition of $0.1 \mathrm{~N}^{\mathrm{AgNO}_{3}}$ into the filtered liquid (based on whether a white precipitation of $\mathrm{AgCl}$ appeared or not). The filtered cake was freeze-dried for 12-18 hours to yield BAPSmodified clay (MMT-BAPS) used in this study.

\subsection{Polyimide matrices}

Poly(amic acid) (PAA) of poly(pyromellitic dianhydride-co-4,4'-oxydianiline) (PM) was supplied by Sigma-Aldrich. The PAA-PM is a 15.0$16.0 \mathrm{wt} \%$ solution in $\mathrm{N}$-methyl-2-pyrrolidone (NMP) with a viscosity of 50-70 poise. PI type films (30-40 $\mu \mathrm{m}$ thick) were prepared from the PAA-PM by casting on soda lime glass plates and subsequent curing in an oven under air atmosphere. Imidization was achieved by placing the films in an air oven for curing at $100^{\circ} \mathrm{C} / 1 \mathrm{hr}, 200^{\circ} \mathrm{C} / 1 \mathrm{hr}$, $300^{\circ} \mathrm{C} / 1 \mathrm{hr}$, and $350^{\circ} \mathrm{C} / 30 \mathrm{~min}$. Subsequently, the cast films were removed after complete imidization from the glass plates by soaking in water. The density of the PI type film prepared from PAA-PM is $1.42 \mathrm{~g} / \mathrm{cm}^{3}$, and the film is hereinafter referred to as PI-PM.

The oligoimide (OI) based on 1,3-bis (3',4,-dicarboxyphenoxy)benzene and 4,4'bis(4"-aminophenoxy)diphenylsulfone and endcapped with phthalic anhydride was synthesized following the procedure previously described in [12]. Note that the oligoimide with the chemical structure shown in Figure 4. was used as a model material for evaluating the rheological behavior of polyimide matrix filled with the nanoparticles.

The number and weight average molecular weights $\left(M_{n}=3609 \mathrm{~g} / \mathrm{mole}\right.$ and $M_{w}=11553 \mathrm{~g} / \mathrm{mole}$ respectively) were obtained from gel permeation chromatography following standard procedures.

\subsection{Processing of polyimide nanocomposites}

The PI-PM nanocomposite films with different concentrations of nanoparticles (i.e., $\mathrm{SNT}, \mathrm{ZrO}_{2}$ or MMT-BAPS) were prepared by adding the desired amount of the nanoparticles to NMP. The resulting suspension of particles in NMP was homogenized with the aid of an ultrasonic mixer $(40 \mathrm{kHz}$, average sonic power: $45 \mathrm{~W}$ ) for 1 hour. The sonicated suspension was transferred into a three neck round bottom flask equipped with a mechanical stirrer, a nitrogen gas inlet, and a drying tube outlet filled with calcium sulfate. After stirring the nanoparticle solution for $10 \mathrm{~min}$, the PAA-PM was added to the nanoparticle suspension and the stirring of the mixture was continued for an additional 60 min until a constant viscosity was obtained. The solid content of the nanoparticles/PAA-PM was $10 \mathrm{wt} \%$ in NMP. Thin (30-40 $\mu \mathrm{m}$ thick) PI-PM nanocomposite films were prepared with varying nanoparticles weight concentrations (wt) from the nanoparticles/PAAPM solution as already described above for the pure (unfilled) PI-PM films. Using the material densities $\left(1.42 \mathrm{~g} / \mathrm{cm}^{3}\right.$ for PI-PM, $2.5 \mathrm{~g} / \mathrm{cm}^{3}$ for SNT, $6 \mathrm{~g} / \mathrm{m}^{3}$ for $\mathrm{ZrO}_{2}$, and $\sim 2 \mathrm{~g} / \mathrm{cm}^{3}$ for MMTBAPS), the corresponding volume concentrations (vol) of nanoparticles in the polyimide nanocomposites were estimated using Equation (1):

$$
v o l=\frac{1}{1+\frac{\rho_{1}}{\rho_{2}} \cdot\left(\frac{1}{w t}\right)} \cdot 100
$$

where $\rho_{1}$ is density of the particles and $\rho_{2}$ is density of the polymer matrix.

A simple solution mixing method was used to prepare a mixture of NMP solution of OI with nanoparticles (i.e., SNT, $\mathrm{ZrO}_{2}$ or MMT-BAPS). Firstly, the nanoparticles were dispersed in NMP using an ultrasonic mixer $(40 \mathrm{kHz}$, average sonic power: $45 \mathrm{~W}$ ) for 1 hour. Various amounts of the nanoparticles were used to yield final OI/nanoparticles mixtures containing 3-20 wt $\%$ of the respective nanoparticles. Subsequently, NMP solution of OI (20 wt $\%$ OI concentration) was added to the modified nanoparticles/NMP dispersion, and the com-

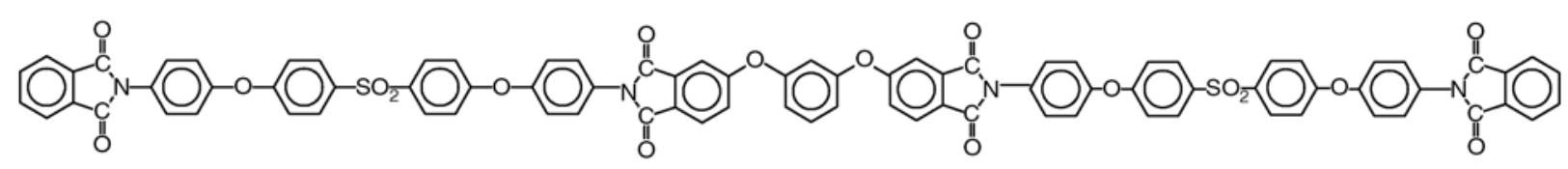

Figure 4. Structure of the oligoimide used to evaluate the rheological behavior 
bined mixture was stirred with a magnetic stirrer for 5 hours followed by ultrasonic mixing of same for 1 hour. The resulting NMP dispersion of OI with the nanoparticles was poured on a Teflon ${ }^{\circledR}$ coated metal substrate that was subsequently heated at $100^{\circ} \mathrm{C}$ for 1 hour, $200^{\circ} \mathrm{C}$ for 1 hour, and $280^{\circ} \mathrm{C}$ for 0.5 hour to completely remove NMP solvent as monitored by TGA. The Teflon ${ }^{\circledR}$ coating made it easy to collect the OI/nanoparticles blends in the form of powder. As described in the next section, these powdered samples were used to prepare test specimens for the rheology measurements described in the next section.

\subsection{Measurements}

A strain-controlled dynamic rheometer ARES ${ }^{\circledR}$ from TA Instruments was used to measure the dynamic and steady shear viscosity of the polymers in the cone and plate configuration following standard procedures. The diameter of the plate was $25 \mathrm{~mm}$ and the cone angle was $0.1 \mathrm{rad}$. Nitrogen was used as the heating gas for temperature control. The powdered samples were compacted into disks at room temperature using a compression molding machine. The disk was then subsequently placed between the plates of the rheometer that were preheated to the desired temperature.

Tensile stress-strain properties of the PI-PM based nanocomposite films were measured using a tensile testing machine (Alliance RT/10, MTS systems Co., Ltd.) following standard ASTM D882-95 method. A specimen gage length of $50 \mathrm{~mm}$ and width of $5 \mathrm{~mm}$ and a testing speed of $2 \mathrm{~mm} / \mathrm{min}$ were used. Care was taken to ensure proper alignment of the specimen with the testing fixture. An abrasive paper was placed between the specimen and test fixture surface to prevent slippage. Ten replicate measurements were conducted for each sample and the average results of the ten specimens are reported in this article.

The XRD patterns of nanocomposites PI-PM/ MMT-BAPS and OI/MMT-BAPS were recorded with an X-ray diffractometer (D/MAX Ultema III, Rigaku Co., Ltd.). The X-ray was generated with nickel-filtered $\mathrm{CuK}_{\alpha}(\lambda=0.154 \mathrm{~nm})$ at $40 \mathrm{kV}$ and $44 \mathrm{~mA}$. The samples were scanned at $2 \theta$ angles ranging from 2 to $10^{\circ}$ using a scanning rate of $10 \%$ min at room temperature. An increase in the basal layer spacing of organically modified MMTs in the PI/MMT composites, which was determined from a shift in the (001) peak position, confirmed ion exchange or polymer intercalation between the silicate layers.

\section{Results and discussion}

\subsection{Rheology of model Oligoimide Based Nanocomposites}

To evaluate the feasibility of homogeneous dispersion of the nanoparticles in polyimide matrix, we used the rheology method that was previously reported to be an effective method for characterizing the exfoliation of clay nanoparticles in a variety of polymers $[12,13]$. Homogenous dispersion of nanoparticles in the polymer should lead to a timedependent rheological behavior (i.e., thixotropy) that is characteristic of formation of percolation type network at some concentration of the nanoparticles. Generally, it is possible to estimate this percolation threshold $P_{c}$ theoretically [14] by using Equation (2) for a cylinder:

$P_{c}=\frac{0,6}{r}$

and Equation (3) for an ellipsoid:

$P_{c}=\frac{1,27}{r}$

where aspect ratio $r=L / d$, and $L$ is the length of cylinder or diameter of ellipsoid and $d$ is the diameter of cylinder or thickness of the ellipsoid. The preceding equations and the average aspect ratio of $\sim 10$ for SNT (Figure 1c) were used to estimate the percolation threshold $\left(P_{c}\right)$ and the $P_{c}(\mathrm{SNT})$ was found to be $\sim 6 \mathrm{vol} \%$. Assuming an average diameter (lateral dimension) of $\sim 200 \mathrm{~nm}$ for the MMT particles [15] and a thickness of $\sim 1 \mathrm{~nm}$ the percolation threshold for the MMT particles was estimated as $P_{c}(\mathrm{MMT})=0.64 \mathrm{vol} \%$.

As previously reported for oligoimides melt blended with clay particles (MMT-15A, Southern Clay Products) [12], the application of strong shear flow field in a HAAKE MiniLab ${ }^{\circledR}$ Micro Compounder can lead to relatively good dispersion of MMT-15A particles in a polymer volume. In addition, the significant viscosity increase exhibited by the OI/MMT nanocomposites after application of 


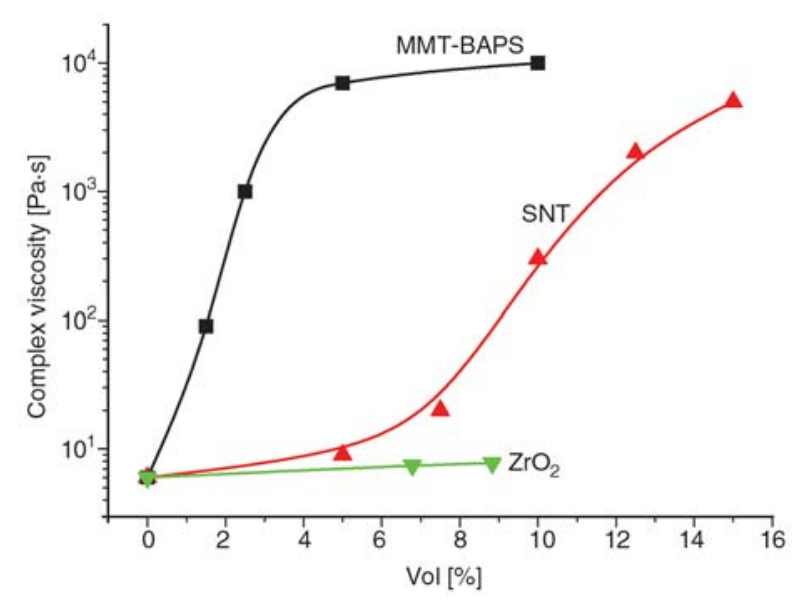

Figure 5. Dependence of complex viscosity on volume concentration of nanoparticles in oligoimide OI. Temperature $T=260^{\circ} \mathrm{C}$, frequency $\omega=1 \mathrm{rad} / \mathrm{s}$, strain $\varepsilon=1 \%$.

the strong shear flow fields may be attributed to the partial exfoliation of the organo-MMT particles and formation of the percolating (network) structure at $\sim 10 \mathrm{wt} \%$ of MMT-15A particles in OI melt as previously reported $[12,13]$. This prior work suggests that OI/MMT clay nanocomposites can be used as excellent model systems for investigating the process of exfoliation (or optimal dispersion) of the MMT-BAPS particles in PI reported in the present paper.

Figure 5 shows the dependence of complex viscosity (frequency $\omega=1 \mathrm{rad} / \mathrm{s}$, strain $\varepsilon=1 \%$ ) on concentration of the nanofillers (i.e., MMT-BAPS, SNT and $\mathrm{ZrO}_{2}$ ) for the present OI nanocomposites prepared as already described in the experimental section 2.6. The viscosities of the nanocomposites were estimated at $260^{\circ} \mathrm{C}$, a temperature corresponding to the fluid state of the OIs. The figure shows significant increase ( $\sim 3$ decades) for the OI/MMTBAPS and OI/SNT nanocomposites occurring at 2-3 vol\% MMT-BAPS and 8-12 vol\% SNT nanofiller concentrations, respectively. For the two types of nanocomposites just mentioned, the critical nanofiller concentrations corresponding to the dramatic rise in viscosity were found to be both higher than the percolation threshold values estimated theoretically as already described (i.e., $P_{c}(\mathrm{MMT})=0.64 \mathrm{vol} \%$ and $\left.P_{c}(\mathrm{SNT})=6 \mathrm{vol} \%\right)$. This trend in the viscosity data is consistent with that previously reported in the literature for similar polymer nancomposites and has been attributed to different degrees of exfoliation of the nanofillers in the polymer matrix $[2,3,12]$. The X-ray data for

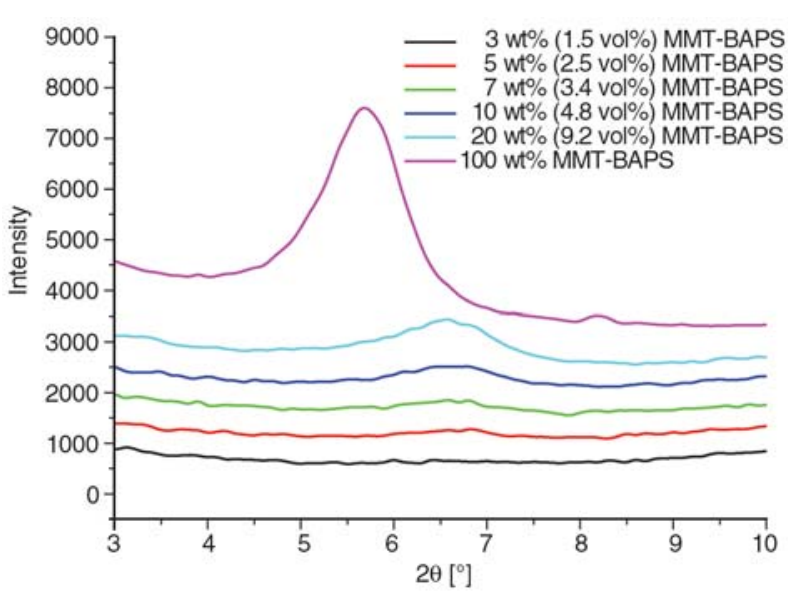

Figure 6. XRD patterns of oligoimide (OI) nanocomposites filled with MMT-BAPS particles. The weight and volume concentrations of the particles are indicated on the graph.

OI/MMT-BAPS nanocomposites at different concentrations of particles (see Figure 6) provide indirect confirmation of this hypothesis because an increase in MMT-BAPS concentration leads to aggregation of the MMT platelets at concentrations $>3 \mathrm{vol} \%$, that is clearly evident from appearance of a diffraction peak at around $2 \theta=6.3^{\circ}$. It is worthy to note that this $2 \theta$ value is equivalent to MMT-BAPS interlayer spacing of $1.4 \mathrm{~nm}$ while the diffraction peak at $2 \theta=7.3^{\circ}$ corresponding to an interlayer spacing of $1.2 \mathrm{~nm}$ (Figure 6).

The preceding results are consistent with our expectations of strong influence of the particle morphology and aspect ratio of the nanofillers (i.e., MMT platelets or SNT cylinders). For example, the relatively low aspect ratio SNT was found to exhibit a dramatic viscosity rise that is known to be consistent with formation of a percolation network structure at relatively higher concentrations than that exhibited by the relatively higher aspect ratio MMT (Figure 5).

As can be seen in Figure 5, the concentration dependence of viscosity for the $\mathrm{OI} / \mathrm{ZrO}_{2}$ nanocomposite was found to be consistent with that predicted by the following classical Thomas equation (4) $[16]$ :

$\eta=1+2.5 \phi+0.05 \phi^{2}+A \exp (B \phi)$

where $\phi$ is volume fraction of particles; $A=0.0273$ and $B=16.6$. In this case, this relatively modest increase in viscosity with increasing $\phi$ (up to $9 \mathrm{vol} \%$ as depicted in Figure 5) is somewhat similar 
to that reported in the literature for polymers filled with spherical particles with various sizes [16]. The observed behavior of the OI/SNT and OI/MMT nanocomposites suggests that anisotropic particles such as MMT or SNT are more effective in inducing formation of percolating network structure (i.e., gelation) than spherical particles such as $\mathrm{ZrO}_{2}$ used in this study [17].

\subsection{Mechanical properties of PI-PM nanocomposite films}

The mechanical properties (Young's modulus $E_{t}$, tensile strength $\sigma_{t}$ and elongation at break $\varepsilon_{t}$ ) of the PI-PM based nanocomposite films are summarized in Table 1. Clearly, this table shows that incorporation of SNT and MMT-BAPS into polyimide matrix improves its modulus considerably. Figure 7 compares the nanofiller concentration dependencies of the relative modulus (i.e., modulus $E_{t}$ divided by the modulus of the pure PI-PM matrix $\left.\left(E_{0}=2.8 \mathrm{GPa}\right)\right)$ for the three types of nanocomposites studied. It is evident from this figure that the PI-PM/MMT-BAPS showed the largest increase in modulus with increasing nanofiller concentration. This finding is consistent with the nanofiller concentration dependencies of the viscosity of the nanocomposites already discussed (see Figure 5). As before, the large increase in modulus just mentioned is thought to be due to the relative high aspect ratios and enhanced dispersion of the MMTBAPS nanofiller compared with that of the isometric $\mathrm{ZrO}_{2}$ nanofillers. The results confirm our expectation that the morphology of the nanoparticles (platelets, tubes or isometric form) and especially their aspect ratios strongly influences the viscoelastic properties of the nanocomposites in both their

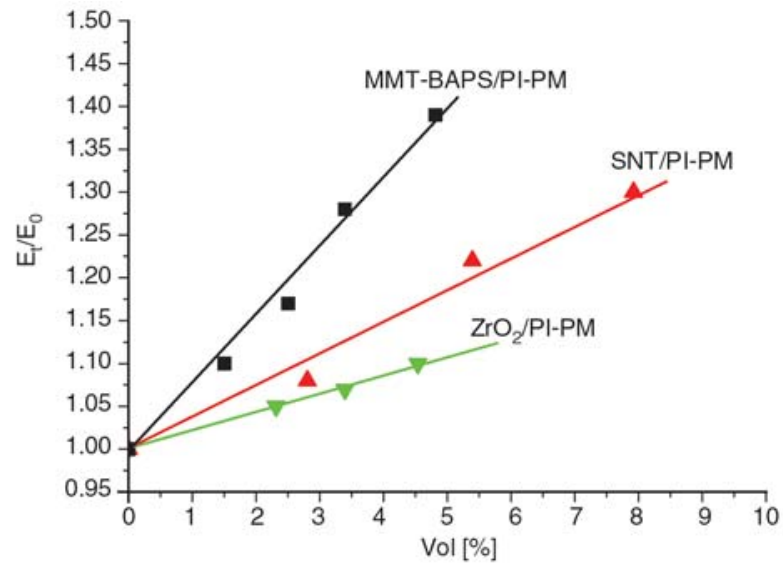

Figure 7. Dependence of tensile modulus $E_{t}$ of nanocomposite films PI-PM on volume concentration of the nanoparticles (see Table $1, E_{0}$ is the modulus of unfilled PI-PM film)

solid and liquid states. Therefore, understanding the role of the nanofiller variables such as morphology, aspect ratio and composition on the properties of polymer nancomposites may lead to development of new materials with optimal properties for targeted application areas.

As shown in Table 1, the tensile strength and deformation-at-break of the PI-PM based nanocomposite films decreases slightly with increasing concentrations of the nanofiller used to an extent that depends on the specific characteristics of the nanofiller used. This decrease in tensile strength may be attributed to the less than optimum adhesion between the nanofillers and the matrix and the possible formation of an inhomogeneous network structure density in the nanocomposite like others have reported for other polymer nanocomposites $[2,3]$. It is worthy to note that formation of an inhomogeneous network structure just mentioned is exacerbated by the strong rise in viscosity near the

Table 1. Properties of nanocomposite films based on PI-PM type polyimide matrix

\begin{tabular}{|c|c|c|c|c|c|}
\hline Particles & $\begin{array}{c}\text { Nanoparticles } \\
\text { concentration [wt \%] }\end{array}$ & $\begin{array}{c}\text { Nanoparticles } \\
\text { concentration [vol\%] }\end{array}$ & $\begin{array}{c}\text { Tensile modulus } \\
{[\mathrm{GPa}]}\end{array}$ & $\begin{array}{c}\text { Tensile strength } \\
\text { [MPa] }\end{array}$ & $\begin{array}{c}\text { Deformation at } \\
\text { break [\%] }\end{array}$ \\
\hline \multirow{5}{*}{ MMT-BAPS } & 0 & 0 & $2.85 \pm 0.05$ & $131 \pm 3$ & $30 \pm 2$ \\
\hline & 3 & 1.5 & $3.13 \pm 0.04$ & $120 \pm 2$ & $22 \pm 6$ \\
\hline & 5 & 2.5 & $3.34 \pm 0.11$ & $117 \pm 5$ & $16 \pm 3$ \\
\hline & 7 & 3.4 & $3.65 \pm 0.07$ & $110 \pm 4$ & $13 \pm 3$ \\
\hline & 10 & 4.8 & $3.96 \pm 0.10$ & $108 \pm 6$ & $5 \pm 2$ \\
\hline \multirow{3}{*}{ SNT } & 5 & 2.8 & $3.07 \pm 0.07$ & $123 \pm 3$ & $24 \pm 2$ \\
\hline & 10 & 5.4 & $3.50 \pm 0.10$ & $97 \pm 2$ & $12 \pm 3$ \\
\hline & 15 & 7.9 & $3.70 \pm 0.10$ & $86 \pm 3$ & $6 \pm 2$ \\
\hline \multirow{3}{*}{$\mathrm{ZrO}_{2}$} & 10 & 2.3 & $3.00 \pm 0.05$ & $105 \pm 2$ & $15 \pm 2$ \\
\hline & 15 & 3.4 & $3.06 \pm 0.07$ & $95 \pm 4$ & $10 \pm 5$ \\
\hline & 20 & 4.5 & $3.13 \pm 0.10$ & $90 \pm 3$ & $7 \pm 3$ \\
\hline
\end{tabular}


percolation threshold as already mentioned, making it crucial to determine as in this study the optimal preparation conditions that may reduce or eliminate any potential micro-heterogeneity in the resultant nanocomposite films. Despite the reduction of the tensile strength caused by incorporation of the nanofillers in the polyimide nanocomposites, it is interesting to note that incorporation of relatively high concentrations of SNT and $\mathrm{ZrO}_{2}$ (up to $10 \mathrm{vol} \%$ ) does not lead to catastrophic decrease of elongation-at-break $\left(\varepsilon_{t}\right)$ that is widely reported in the literature for a number of PI nanocomposites containing organoclay nanofillers $[2,3]$. This last point suggests the possibility of using the present SNT and $\mathrm{ZrO}_{2}$ to develop useful polyimide nanocomposites films with improved thermal and barrier properties, and adequate mechanical properties for a number of coating applications where the coating film flexibility is an important performance requirement. This important benefit offered by the SNT and $\mathrm{ZrO}_{2}$ is believed to be due to the desirable intrinsic properties of these ceramic particles that can be prepared with prescribed properties for specific applications including polymer nanocomposites as already mentioned. This study may stimulate a better understanding of the effects of methods of incorporation of nanoparticles with varying shapes and sizes into polyimide matrices and the effects of the nanoparticles morphology on the structure and properties of polyimide nanocomposites, enhancing our ability to prepare useful polymer nanocomposites in general with improved properties for targeted applications where common polymer nanocomposite systems are not useable.

\section{Conclusions and outlook}

It can be concluded from the results of this study that simple rheometric methods can be reliably used to probe the influence of methods of incorporation of nanoparticles with varying shapes and sizes into polyimide matrices and the effects of the nanoparticles morphology on the quality of dispersion and properties of polyimide nanocomposites. By using model oligimides systems filled with the three types of nanoparticles studied (i.e., MMTBAPS, SNT and $\mathrm{ZrO}_{2}$ ), it was demonstrated that the critical concentration for gelation or formation of a percolating network structure that is characteristic of the huge rise in viscosity with increasing nanoparticles concentration strongly depends on the morphology and aspect ratio of the nanoparticles. The significant increase in viscosity ( $\sim 3$ decades) exhibited by the model oligoimides nanocomposites filled with MMT-BAPS, SNT or $\mathrm{ZrO}_{2}$ nanofillers was observed at 2-3 vol\% MMTBAPS and 8-12 vol\% SNT or $\mathrm{ZrO}_{2}$ nanofiller concentrations, respectively.

On the other hand, the critical nanofiller volume concentrations corresponding to the dramatic rise in viscosity for the OI/MMT-BAPS and OI/SNT nanocomposites were found to be higher than the percolation threshold values $\left(P_{c}\right)$ estimated theoretically (i.e., $P_{c}(\mathrm{MMT})=0.64 \mathrm{vol} \%$ and $P_{c}(\mathrm{SNT})=$ 6 vol\%). This disagreement between the experimental and theoretical estimations of $P_{c}$ is thought to be due to agglomeration of the nanoparticles that was indirectly confirmed by XRD data for the OI/MMT nanocomposite.

In general, it is worthy to note that the SNT and $\mathrm{ZrO}_{2}$ nanoparticles of this study can be used to develop useful polyimide nanocomposites films with adequate thermo-mechanical properties for a number of coating applications where the coating film flexibility and thermooxidative stability are important performance requirements. Overall, the results confirm our expectation that the morphology of the nanoparticles (platelets, tubes or isometric form) and especially their aspect ratios strongly influences the viscoelastic properties of the nanocomposites in both their solid and liquid states.

\section{Acknowledgements}

Financial support of this work by the U.S. National Science Foundation under contract grant numbers CBET 0317646 and CBET 0752150, and by the Russian Foundation of Basic Research under contract grant number 07-03-00846-a is gratefully acknowledged.

\section{References}

[1] Xanthos M.: Functional fillers for plastics. WileyVCH, Weinheim (2005).

[2] Sinha Ray S., Okamoto M.: Polymer/layered silicate nanocomposites: A review from preparation to processing. Progress in Polymer Science, 28, 1539-1641 (2003).

[3] Zeng Q., Yu A., Lu G., Paul D.: Clay-based polymer nanocomposites: Research and commercial development. Journal of Nanoscience and Nanotechnology, $\mathbf{5}$, 1574-1592 (2005) 
[4] Kashiwagi T., Du F., Douglas J. F., Winey K. I., Harris R. H., Shields J. R.: Nanoparticle networks reduce the flammability of polymer nanocomposites. Nature Materials, 4, 928-932 (2005).

[5] Falini G., Foresti E., Lesci G., Roveri N.: Structural and morphological characterization of synthetic chrysotile single crystals. Chemical Communication, 2002, 1512-1513 (2002).

[6] Zhang Y., Lu S., Li Y., Dang Z., Xin J., Fu S., Li G., Guo R., Li L.: Novel silica tube/polyimide composite films with variable low dielectric constant. Advance Materials, 17, 1056-1059 (2005).

[7] Korytkova E., Maslov A., Pivovarova L., Drozdova I., Gusarov V.: Formation of $\mathrm{Mg}_{3} \mathrm{Si}_{2} \mathrm{O}_{5}(\mathrm{OH})_{4}$ nanotubes under hydrothermal conditions. Glass Physics and Chemistry, 30, 51-55 (2004).

[8] Pozhidaeva O., Korytkova E., Romanov D., Gusarov $\mathrm{V}$.: Formation of $\mathrm{ZrO}_{2}$ nanocrystals in hydrothermal media of various chemical compositions. Russian Journal of General Chemistry, 72, 849-853 (2002).

[9] Yudin V., Otaigbe J., Gladchenko S., Olson B., Nazarenko S., Korytkova E., Gusarov V.: New polyimide nanocomposites based on silicate type nanotubes: Dispersion, processing and properties. Polymer, 48, 1306-1315 (2007).
[10] Bessonov M., Koton M., Kudryavtsev V., Laius L. A.: Polyimides-thermally stable polymers. Consultants Bureau, New York (1987).

[11] Gelest, Inc, Edited by B. Arkles, 2nd edition, 88-89 (1998).

[12] Yudin V., Divoux G., Otaigbe J., Svetlichnyi V.: Synthesis and rheological properties of oligoimide/montmorillonite nanocomposites. Polymer, 46, 1086610872 (2005).

[13] Zhong Y., Wang S-Q.: Exfoliation and yield behavior in nanodispersions of organically modified montmorillonite clay. Journal of Rheology, 47, 483-495 (2003).

[14] Garboczi E., Snyder K., Douglas J., Thorpe M.: Geometrical percolation threshold of overlapping ellipsoids. Physical Review E, 52, 819-828 (1995).

[15] Giannelis E., Krishnamoorti R., Manias E.: Polymersilicate nanocomposites: Model systems for confined polymers and polymer brushes. Advances in Polymer Science, 138, 107-120 (1999).

[16] Nielsen L.: Mechanical properties of polymers and composites. Marcel Dekker, New York (1994).

[17] Barnes H.: Thixotropy- A review. Journal of NonNewtonian Fluid Mechanics, 70, 1-33 (1997). 